\title{
Minimally Invasive Surgery in Loss of the Guide Wire: Case Report
}

\author{
Godratollah Maddah ${ }^{1}$, Abbas Abdollahi ${ }^{2}$, Ali Jangjoo ${ }^{2}$, Sadjad Noorshafiee ${ }^{1}$, Mohsen Ab- \\ dollahi ${ }^{2}$, Ali Mohammad Hasanzadeh ${ }^{3}$ \\ ${ }^{1}$ Endoscopic and Minimally Invasive Surgery Research Center, Ghaem Hospital, Faculty of Medicine, Mashhad University of Medical Sciences, Mashhad, IR Iran \\ ${ }^{2}$ Surgical Oncology Research Center, Imam Reza Hospital, Faculty of Medicine, Mashhad University of Medical Sciences, Mashhad, IR Iran \\ ${ }^{3}$ Faculty of Nursing and Midwifery, Mashhad University of Medical Sciences, Mashhad, IR Iran
}

\begin{tabular}{l}
\hline A R T I C L E I N F O \\
\hline Article type: \\
Case Report \\
\hline Article history: \\
Received:12 Dec 2011 \\
Accepted: 05 May 2012 \\
Revised: 27 July 2012
\end{tabular}

\begin{abstract}
A B S T R A C T
Background: Catheterization of central veins is a routine technique which is widely used in general hospitals and medical intensive care units. It should be carefully performed and managed to prevent adverse side effects.

Case Presentation: In this case report, we describe a case of lost guide wire during central venous catheterization, which was successfully treated with a minimally invasive surgical technique.

Conclusions: Inattention is the main cause of the retained guide wires. The interventional angiography is usually successful as the first line therapy.
\end{abstract}

Keywords:

Catheters

Catheterization

Central Venous

Surgical Procedures

Minimally Invasive

Complications

Implication for health policy/practice/research/medical education:

Loss of the guide wire is a rare, relatively dangerous, and preventable complication. Inattention is the main cause of the retained guide wires. The diagnosis is simple, but they are usually disregarded by the radiologists. The guide wires should be removed as quickly and completely as possible to prevent any complication. The interventional angiography is usually successful as the first line therapy.

- Please cite this paper as:

Maddah Gh, Abdollahi A, Jangjoo A, Noorshafiee S, Abdollahi M, Hasanzadeh AM. Minimally Invasive Surgery in Loss of the Guide Wire: Case Report. J Minim Invasive Surg Sci. 2013; 2(1):108-10. DOI: 10.5812/jmiss.3795

\section{Background}

Central venous catheterization is a routine technique in modern medicine. Central venous catheters are needed for monitoring patients in special conditions and as a route for parenteral nutrition. Depending on the catheter type, cannulation is performed at different sites such as the jugular, subclavian, femoral and brachial veins. Complications associated with the central venous catheters are infection, failure to place the catheter, arterial puncture, improper catheter position, pneumothorax, hematoma, hemothorax, asystolic cardiac arrest of unknown etiology, and inferior vena cava (IVC) trauma (1).

* Corresponding author: Abbas Abdollahi, Surgical Oncology Research Center, Imam Reza Hospital, Faculty of Medicine, Mashhad University of Medical Sciences, Mashhad, IR Iran. Tel:+98-9153141917, Fax:+98-5118022677, E-mail: AbdollahiA@mums.ac.ir

DOI:10.5812/jmiss.3795

Copyright @ 2013, Minimally Invasive Surgery Research Center and Mediterranean \& Middle Eastern Endoscopic Surgery Association. This is an open-access article distributed under the terms of the Creative Commons Attribution License, which permits unrestricted use, distribution, and reproduction in any medium, provided the original work is properly cited. 
Infection is the most common complication in subclavian vein route; though arrhythmias and air embolism can occur (2). These complications occur in about $12 \%$ of cases $(2,3)$. One of these extremely rare complications is intravascular loss of a guide wire, which is usually recognized immediately or sometimes with delay (2-5). Since radiologists are attuned to detecting small and medium size foreign bodies, most are likely unaware that an object as large as $60 \mathrm{~cm}$ in length can be unintentionally misplaced within a patient (5). Here, we elucidate a case of retained guide wire during cannulation of the right internal jugular vein, which was successfully treated with a minimally invasive surgical technique.

\section{Case Presentation}

A 65 year old woman presented to the emergency department with abdominal pain in RUQ, fever, chills, and jaundice. She was hospitalized with primary diagnosis of chollangitis. Because of patient's history of heart disease, the central venous catheterization was attempted for fluid resuscitation via the right internal jugular vein using the Seldinger technique. The catheter was inserted by a first-year surgical resident with no supervision by senior residents. No complications or difficulties in cannulation were reported; back flow was also normal. The catheter tray was not checked for the guide wire after the procedure. After the patient was stabilized, she underwent chest X-ray, plain abdominal radiography, and ultrasonography. The radiographs were assessed by the radiology resident and then re-assessed by the surgical resident. The ultrasonography revealed dilated common bile duct according to paraclinic and liver function tests. So, the definitive diagnosis of chollangitis was established. Fluid therapy and suitable antibiotics were started and the patient became a candidate for endoscopic retrograde cholangiopancreatography (ERCP). One day later, the case was reviewed during the surgical morning report session and the faculty members noticed the guide wire in chest and abdominal radiographs. The radiographs showed that the catheter had been entered in the inferior vena cava (IVC) via the right internal jugular vein and then, had remained in the right iliac vein at the point of bifurcation into the internal and external iliac veins (Figures 1 and 2). Based on the consultation with the vascular surgeons and radiologists, the patient underwent interventional radiology for the removal of the catheter. The patient was heparinized, and the foreign body was caught by a gooseneck snare passed via the femoral vein. The method was successful and the retained guide wire was removed by interventional radiology using endovascular retrieval forceps (gooseneck snare). The day after, the patient underwent ERCP and endoscopic sphincterotomy (ES) for chollangitis. The procedure was successful, but unfortunately the septic course continued and despite aggres-

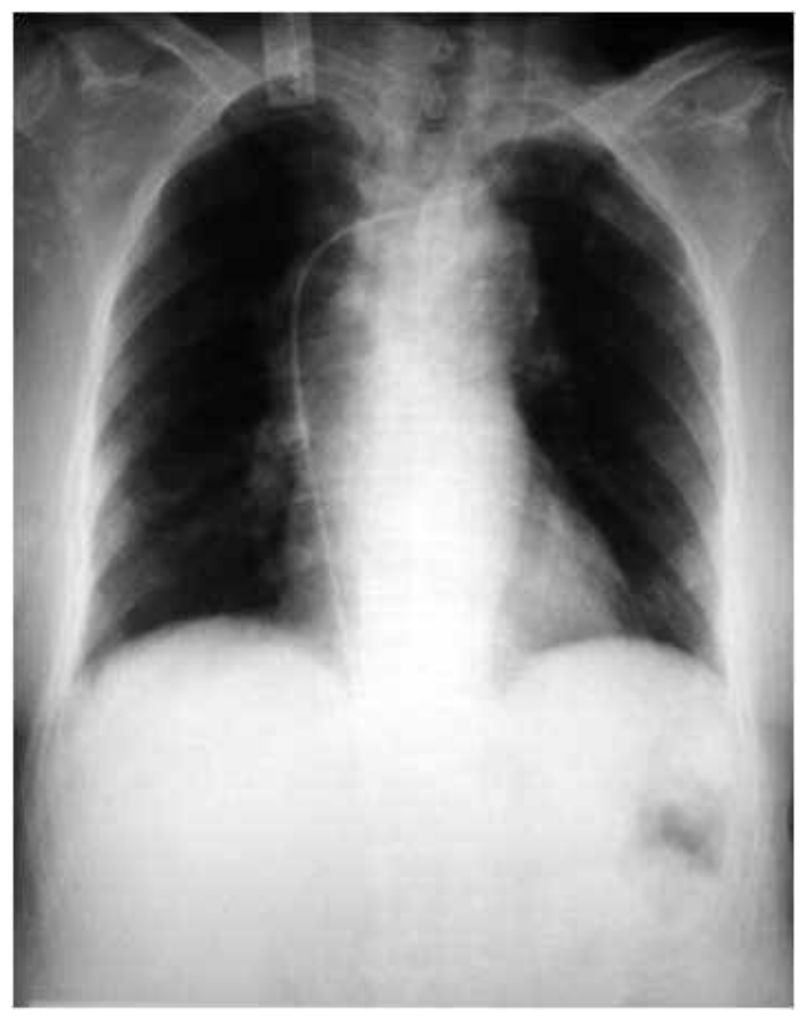

$\overline{\text { Figure 1. The Chest X-Ray Showing the Guide Wire Passed Through the }}$ Internal Jugular Vein to the Inferior Vena Cava

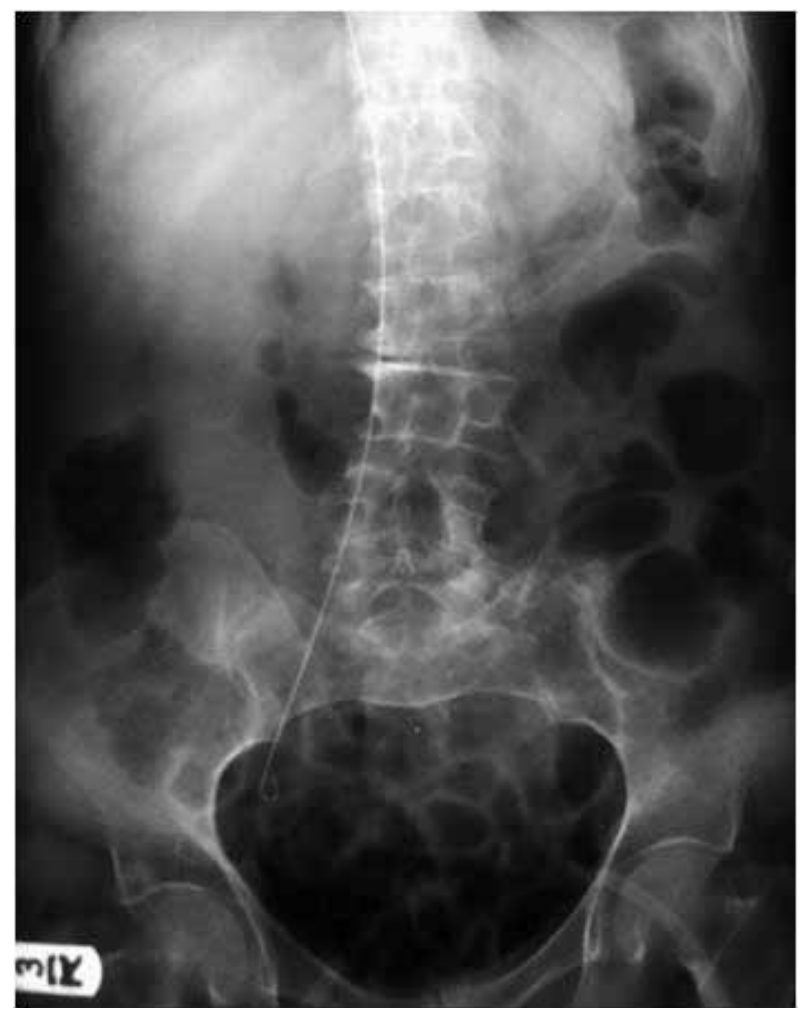

Figure 2. The Abdominal X-Ray Showing the Guide Wire in the Right Common Iliac 
sive treatment, she died with feature of end stage septic shock.

\section{Discussion}

Central venous catheterization has many complications such as infection, failure to place the catheter, arterial puncture, improper catheter position, pneumothorax, hematoma, and so on $(1,2)$. This study reports a case of retained guide wire and its treatment. In our case, the catheter was retained in the right iliac vein at the point of bifurcation. An important point in preventing this complication is that the guide wire should be held at least 18 $\mathrm{cm}$ distant from the vein $(3,5)$. If this rule is followed, the guide wire cannot get lost. Predisposing factors include: inattention, inexperienced operator, inadequate supervision of trainees and exhausted staff $(3,5)$, which all influence our case. The signs of guide wire loss include: missing the guide wire in the tray after the procedure, resistance to injection via catheter, poor venous backflow, guide wire visibility on radiographs, asymptomatic, and deep vein thrombosis $(4,5)$. Our patient was asymptomatic. The diagnosis is very simple, which is often established incidentally during routine radiographic exams $(4,5)$. However, radiologists are often searching for certain routinely detected objects such as the laparotomy pads, reactors, or surgical clamps, but they would never expect for a misplaced guide wire with $60 \mathrm{~cm}$ length (6). Another cause of overlooking the guide wire in radiographs is multiple tubes that are inserted for various reasons such as nasogastric tubes and foley catheters (7, 8). There are limited published literatures on the complications of lost guide wire, but such a foreign body can cause arrhythmias, vascular damage, thrombosis, cardiac tamponade, arterial puncture, and embolism (3-6, 9 ). The potential risk of death or serious complications from retained catheter fragments has been reported as high as $71 \%$ (9). Our patient did not experience any complication from the retained guide wire. There are different methods for the management of the retained guide wires, which the interventional radiology is the method of choice. Usually, the foreign body is caught by modern devices such as the goose-neck snare catheter, endovascular forceps, or dormier basket, which the two latest methods increase the risk of endovascular trauma $(4,5)$. The foreign body should be removed along with the vascular sheath, which is usually twice the size of the lost guide wire. In the case of failure, or when heparinization is contraindicated, extraction should be attempted by careful surgical exploration $(5,6)$. This method was successfully performed in our patient.

\section{Conclusion}

Loss of the guide wire is a rare, relatively dangerous, and preventable complication. Inattention is the main cause of the retained guide wires. The diagnosis is simple, but they are usually disregarded by the radiologists. The guide wires should be removed as quickly and completely as possible to prevent any complication. The interventional angiography is usually successful as the first line therapy.

\section{Acknowledgments}

We sincerely acknowledge Ms. M. Hassanpour for editing the manuscript.

\section{Authors' Contribution}

None declared.

\section{Financial Disclosure}

The authors declare no conflict of interest.

\section{Funding/Support}

None declared.

\section{References}

1. Wolf F, Schernthaner RE, Dirisamer A, Schoder M, Funovics M, Kettenbach J, et al. Endovascular management of lost or misplaced intravascular objects: experiences of 12 years. Cardiovasc Intervent Radiol. 2008;31(3):563-8.

2. Abuhasna S, Abdallah D, Ur Rahman M. The forgotten guide wire: a rare complication of hemodialysis catheter insertion. J Clin Im aging Sci. 2011;1:40.

3. Brunicardi F, Brandt M, Andersen D, Billiar T, Dunn D, Hunter J, et al. Schwartz's Principles of Surgery. New York: McGraw-Hill Prof Med/Tech; 2010. p. 314-342.

4. Guo H, Peng F, Ueda T. Loss of the guide wire: a case report. Circ J. 2006;70(11):1520-2.

5. Schummer W, Schummer C, Gaser E, Bartunek R. Loss of the guide wire: mishap or blunder? BrJ Anaesth. 2002;88(1):144-6.

6. Chong CF. An unexpected image on chest radiograph-dislodged central venous catheter guide wire. Resuscitation. 2006;68(1):162-3.

7. Perez-Diez D, Salgado-Fernandez J, Vazquez-Gonzalez N, Calvino-Santos R, Vazquez-Rodriguez JM, Aldama-Lopez G, et al. Images in cardiovascular medicine. Percutaneous retrieval of a lost guidewire that caused cardiac tamponade. Circulation. 2007;115(24):e629-31.

8. Struck MF, Kaden I, Heiser A, Steen M. Cross-over endovascular retrieval of a lost guide wire from the subclavian vein. J Vasc Access. 2008;9(4):304-6.

9. Cassie CD, Ginsberg MS, Panicek DM. Unsuspected retained 60cm intravenous guidewire. Clin Imaging. 2006;30(4):287-90. 